\title{
Novel Thermodynamic Property and Molecular Chain of Polystyrene Nano-Platelet
}

\author{
By Jiang Jiang GUO* and Xiao Yu LI
}

L-PS was prepared by free-radical polymerization in emulsion with SDS liquid-crystal template. Based on differential scanning calorimetry (DSC), L-PS was found to be different with known PS. As atactic polymer, L-PS displayed one exothermic peak near $150{ }^{\circ} \mathrm{C}$ during the first DSC scan, and the enthalpy of the exothermic peak is large. This is perhaps the first report that atactic polystyrene display this result. From quench process, we found L-PS can form ordered structure under especial temperature. These results could be attributed to novel molecular chain of L-PS.

KEY WORDS: Polystyrene Nanomaterials / Polymer Synthesis / Template / Thermodynamic Property / Molecular Chain /

Different with other material, polymer is composed of many long molecular chains. ${ }^{1}$ For atactic polymer, its molecular chain is random coil mode. ${ }^{2}$ Molecular chain of polymer can be tested by DSC. Different polymer or the same polymer with different molecular chain mode has its unique DSC result. $^{3}$

Nanostructured materials have generated a great deal of interest because of their potential applications. ${ }^{4-7}$ Polymer nanoparticles are important nanostructured materials which can be used as environmentally benign paints and coating. ${ }^{8}$ Usually, polymer nanoparticle prepared by free-radical emulsion polymerization is atactic polymer, which possesses spherical shape. ${ }^{8}$ Molecular chains of these spherical nanoparticles are random coil mode.

Nowadays, a novel polystyrene nano-platelet (L-PS) with lamellar morphology has been synthesized by free radical polymerization in surfactant liquid crystal template. ${ }^{9}$ L-PS had morphology with a very short thickness, and the thickness was shorter than its diameter very much. The especial morphology conflicted with thermodynamic property of random coil molecular chain.

In this paper, we researched novel thermodynamic property and molecular chain mode of L-PS. Moreover, reason which caused the formation of L-PS was also researched.

\section{EXPERIMENTAL}

\section{Materials}

Styrene from Shanghai Gaoqiao Chemical Company was distilled twice under reduced pressure before polymerization. Sodium dodecyl sulfate (SDS) obtained from P. T. Palekao Co. was purified by recrystallization. Potassium persulfate (KPS) from Shanghai First Reagent Plant was purified by crystallization in water. Potassium chloride $(\mathrm{KCl})$ was from Shanghai First Reagent Plant. Deionized water was used for all experiments.

\section{Preparation of L-PS}

In a 500-ml three-necked flask, $40 \mathrm{~mL}$ of deionized water was added to $0.96 \mathrm{~g}$ of $\mathrm{KCl}$ and $1.8 \mathrm{~g}$ of solid SDS. This solution was continuously stirred at $25^{\circ} \mathrm{C}$ until the SDS was fully dissolved. The solution of $3.84 \mathrm{~g}$ of $\mathrm{KCl}$ and $160 \mathrm{~g}$ of deionized water was then added into the previous system and stirred for $0.5 \mathrm{~h}$. The whole process was kept at $50^{\circ} \mathrm{C}$.

Polymerization of polystyrene (PS) was then carried out at $50{ }^{\circ} \mathrm{C}$ and taken $20 \mathrm{~h}$ to complete by starting with the addition of $0.008 \mathrm{~g}$ of initiator ammonium persulfate (APS) and $8 \mathrm{~mL}$ of freshly distilled styrene to the mixture. The crude product was separated from the turbid system by a centrifugal process and then purified by washing with $50 \%$ ethanol-water. The resulting white powder was finally dried in a vacuum oven. During purification, the temperature was always kept below $50{ }^{\circ} \mathrm{C}$ to ensure that the conformation of PS was not changed.

\section{Characterization}

Infrared (IR) spectra were recorded by a Bruker TENSOR $37 \mathrm{FT} / \mathrm{IR}$ using $\mathrm{KBr}$ pellets.

${ }^{13} \mathrm{C}$ NMR spectrum was obtained from a Bruker MSL-300 spectrometer using $\mathrm{CDCl}_{3}$ as the solvent.

Transmission electronic microscopy (TEM) images were obtained on a Tecnai G2 microscope using an acceleration voltage of $120 \mathrm{kV}$. The dry product was resuspended in deionized water, and one drop of the dispersion was allowed to dry on a copper grid which was previously coated with a thin film of carbon deposited by vacuum evaporation.

AFM experiments were performed with a Nanoscope IIIa scanning probe microscope. The height and phase images were obtained simultaneously while operating the instrument. Images were taken at the fundamental resonance frequency of the Si cantilevers which was typically around $300 \mathrm{KHz}$. Phase images represented the variations of relative phase shifts and were thus able to distinguish materials by their property.

Differential scanning calorimetry (DSC) was investigated by using a DERKIN ELMER Pyris I instrument. The measure- 
ments were carried out with a heating rate of $10^{\circ} \mathrm{C} / \mathrm{min}$, while a nitrogen gas purge was used.

X-Ray diffraction (XRD) measurements were performed on a Rigaku $\mathrm{D} / \mathrm{max}-2400$ instrument with $\mathrm{Cu} \mathrm{K} \alpha$ radiation $(\lambda=1.5418 \AA)$.

\section{RESULTS}

The IR spectra of product and ordinary PS were compared in Figure 1, from which no obvious differences with respect to the position and shape of absorption bands could be found. Furthermore, characteristic absorption of SDS (such as 1220 and $985 \mathrm{~cm}^{-1}$ ) was hardly detected in Figure 1a, which means that SDS was almost completely removed from product during purification and product was pure polystyrene.

Figure 2 showed the ${ }^{13} \mathrm{C}$ NMR spectrum of polystyrene at around $145 \mathrm{ppm}$. Here, polystyrene had split absorptions at 144.8-146.2 ppm, which suggested that this polystyrene was essentially atactic. ${ }^{10}$

The transmission electronic microscopy (TEM) observation clearly confirmed that the polystyrene exhibited a layered

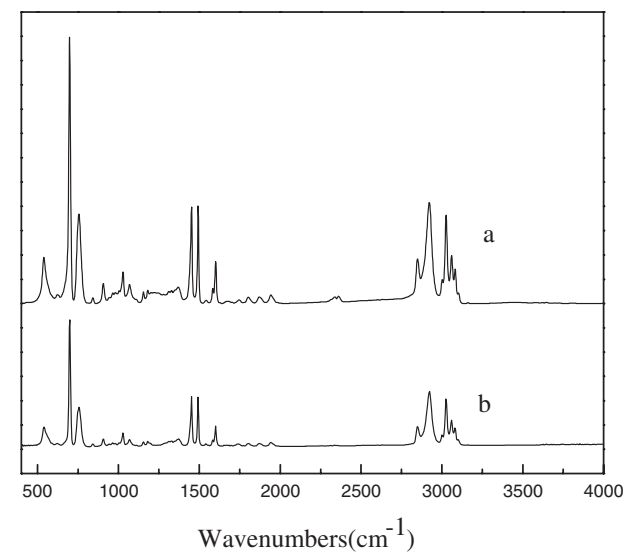

Figure 1. IR spectra of (a) L-PS and (b) ordinary atactic PS.

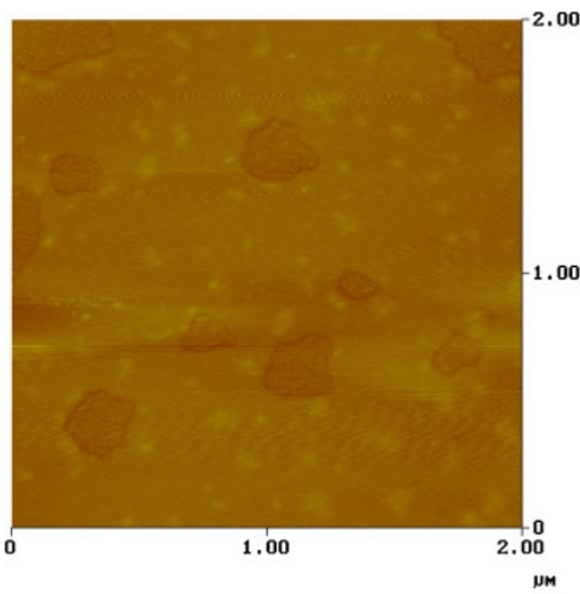

11161503.001 image as presented in Figure 3. Figure 4 showed that the thickness of L-PS was about $3 \mathrm{~nm}$, which is much thinner as compared to an ordinary crystalline lamella $(\sim$ several $10 \mathrm{~nm})$.

Figure 5 showed the DSC curves of L-PS. The sample was scanned to $280{ }^{\circ} \mathrm{C}$ for the first scan, and the result was shown in

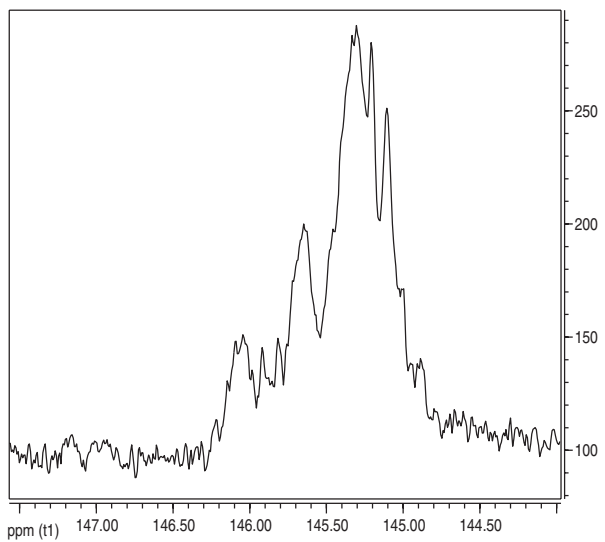

Figure 2. ${ }^{13} \mathrm{C}$ NMR spectra of L-PS.

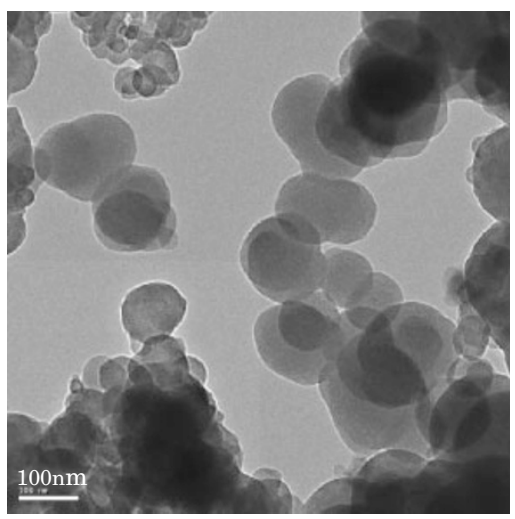

Figure 3. TEM images of L-PS particles.

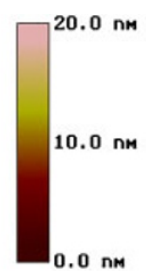

Digital Instruments NanoScope

Scan size $2.000 \mathrm{MM}$

$\begin{array}{ll}\text { Scan size } & 2.000 \\ \text { Scan rate } & 0.7266 \mathrm{~Hz}\end{array}$

Number of samples Height

$\begin{array}{lr}\text { lmage Data } & \text { Height } \\ \text { Data scale } & 20.00 \mathrm{~nm}\end{array}$

Figure 4. Height photo of AFM of L-PS. 


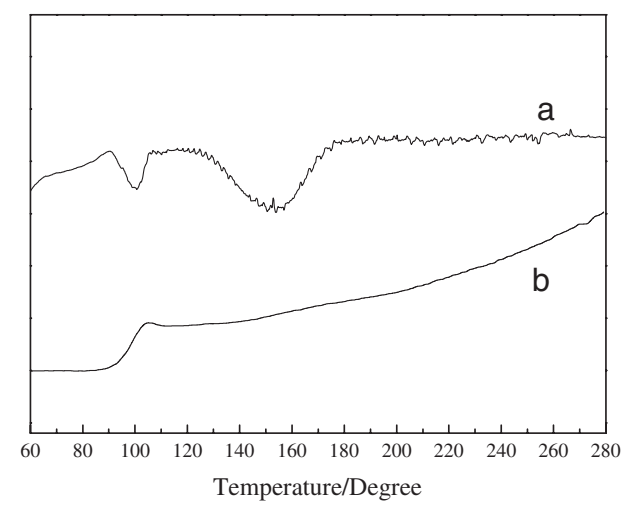

Figure 5. DSC thermograms of L-PS: (a) first scan. (b) second scan.

Figure 5a. After cooling to ambient temperature, the sample was scanned to $280^{\circ} \mathrm{C}$ again, and the result was shown in Figure $5 \mathrm{~b}$. During the first scan, L-PS displayed two exothermic peaks, one at $105^{\circ} \mathrm{C}$, and the other at $154^{\circ} \mathrm{C}$. The subsequent scan after cooling to ambient temperature showed glass transition $\left(T_{\mathrm{g}}\right)$ only, and both exothermic peaks disappeared.

\section{DISCUSSION}

Result of ${ }^{13} \mathrm{C}$ NMR proved that L-PS was atactic polymer. Usually, molecular chain of atactic polymer is random coil mode, which should only demonstrate $T_{\mathrm{g}}$ during the first DSC scan. Novel DSC result of L-PS showed that it should have especial molecular chain mode.

During the first scan, L-PS displayed the first exothermic peak around $T_{\mathrm{g}}\left(100^{\circ} \mathrm{C}\right)$. As to polymer nano-materials, the exothermic peak around $T_{\mathrm{g}}$ may be due to the volume shrinkage of polymer nano-particles, and the loss of surface area results in release of surface free energy. ${ }^{11,12} \mathrm{~L}-\mathrm{PS}$ can be also observed sintering in the heating process. Therefore, the first exothermic peak around $105^{\circ} \mathrm{C}$ was due to the sintering of L-PS particles.

During the first scan, L-PS displayed the second exothermic peak around $154{ }^{\circ} \mathrm{C}$. It was doubted that the second exothermic peak was, perhaps, related to the impurity of L-PS sample, for example, the existence of very little SDS resulting in the above phenomenon. For the sake of insurance, the same styrene emulsion system was polymerized and the compound of L-PS and SDS without purification was scanned on DSC. The result was shown in Figure 6. Moreover, the same emulsion system without styrene was prepared and pure SDS was also scanned on DSC. The result was shown in Figure 7. As can be seen in Figure 6, there was no exothermic peak, but two endothermic peaks in the whole DSC scan for pure SDS. This result had been proven by Fu et al. ${ }^{11}$ For the compound of L-PS and SDS, a compound peak of endothermic peak and exothermic peak near $150{ }^{\circ} \mathrm{C}$ was observed. On the basis of these observations, it is confirmed that the second exothermic peak cannot be attributed to impurity and SDS only resulted in two endothermic peaks from $50{ }^{\circ} \mathrm{C}$ to $200^{\circ} \mathrm{C}$.

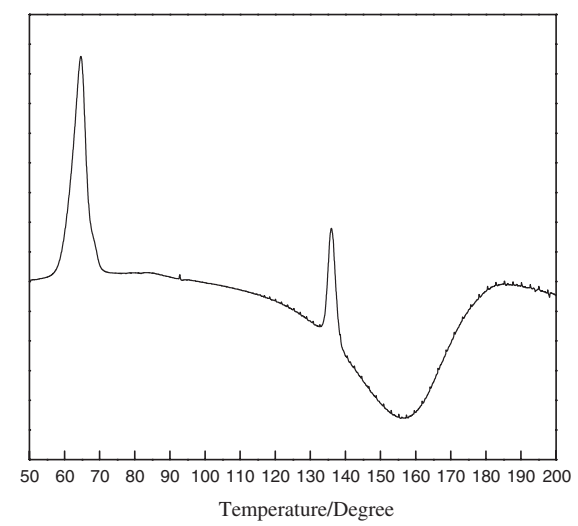

Figure 6. DSC thermograms of the compound of L-PS and SDS.

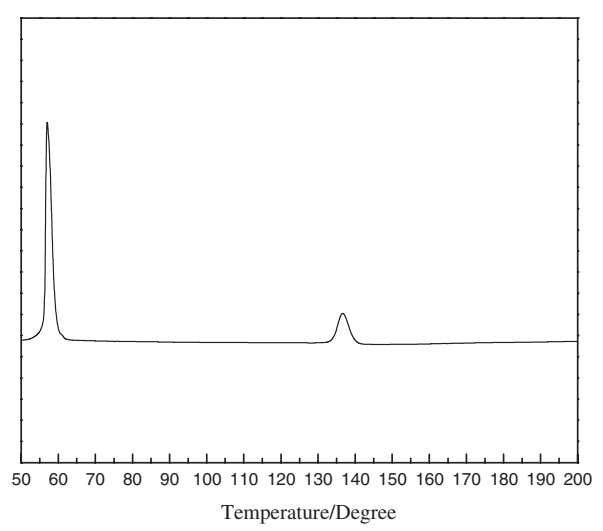

Figure 7. DSC thermograms of pure SDS.

What causes the second exothermic peak? At the beginning, it was considered that the second exothermic peak was due to stereoregulatiry of the polystyrene chain in L-PS, since its position in the DSC curve was just similar to that of isotactic polystyrene (iso-PS) or syndiotactic polystyrene (syn-PS). ${ }^{13}$ However, the result of ${ }^{13} \mathrm{C}$ NMR showed that L-PS was atactic polymer. At the same time, because there was no melting peak in the DSC curve of L-PS, it is not suitable to assume it as a crystallization peak. Even if the molecular chain of atactic polystyrene have crystallizability in a confined space in L-PS, the second exothermic peak should not be thought as a crystallization peak because no perfect crystalline formed. At the beginning of the course of the heating, the ordered region of L-PS formed within the confined space. However, it gradually grows and become larger. Once the ordered structure region grows larger, the spatial confinement is no more effective. Ordered structure of the L-PS become unstable because the atactic polystyrene has intrinsically no crystallizability. Therefore, we would prefer to attribute this exothermic peak to the formation of some kinds of ordered structure in the confined space in L-PS, as discussed below.

L-PS was heated to some designated temperatures and then quenched quickly into liquid nitrogen. The obtained L-PS was examined on a wide-angle X-ray diffractometer. Wide-angle $\mathrm{X}$-ray diffraction patterns are shown in Figure 8. The virgin L- 


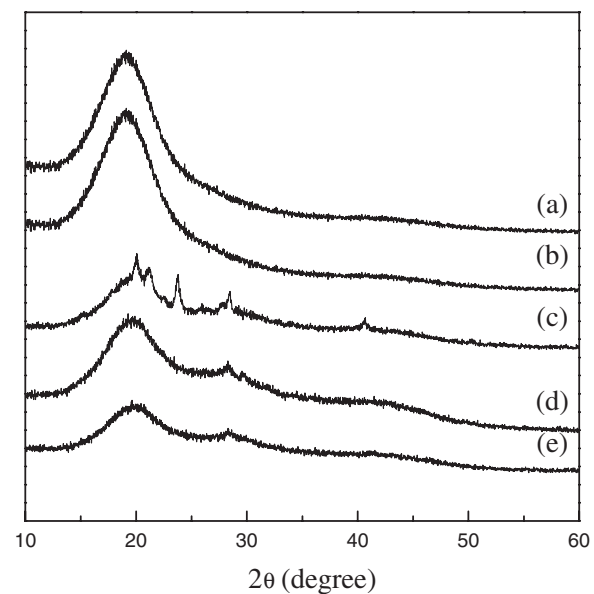

Figure 8. WAXD patterns of L-PS: (a) original L-PS, (b) quenched at $105^{\circ} \mathrm{C}$, (c) quenched at $150^{\circ} \mathrm{C}$, (d) quenched at $180^{\circ} \mathrm{C}$, (e) quenched at $150^{\circ} \mathrm{C}$ for $10 \mathrm{~min}$.

PS sample demonstrated a broad noncrystalline peak at around $20^{\circ}$ (Figure $8 \mathrm{a}$ ), much like ordinary PS prepared by conventional emulsion polymerization. When L-PS was heated to $105^{\circ} \mathrm{C}$, i.e., after the occurrence of the first exothermic peak, and then quenched into liquid nitrogen, similar broad noncrystalline peak appeared (Figure 8b). When L-PS was heated to $150{ }^{\circ} \mathrm{C}$, i.e., after the occurrence of the second exothermic peak, and then quenched into liquid nitrogen, new peaks appeared at $2 \theta$ of $23.8^{\circ}, 28.5^{\circ}$ and $40.6^{\circ}$ (Figure $8 \mathrm{c}$ ). When L-PS was heated at $180^{\circ} \mathrm{C}$ and then quenched into liquid nitrogen, new peaks all almost disappeared and the broad peak around $20^{\circ}$ recovered (Figure $8 \mathrm{~d}$ ). When the sample was annealed at $150{ }^{\circ} \mathrm{C}$ for $10 \mathrm{~min}$ and then quenched into liquid nitrogen, its WAXD spectrum (shown in Figure 8e) is similar to Figure $8 d$.

From the result of Figure $8 \mathrm{~b}$, it can be concluded that the first exothermic peak around $105^{\circ} \mathrm{C}$ could not be caused by change of molecular chain mobility; otherwise, the WAXD pattern of Figure $8 \mathrm{~b}$ would be different with Figure 8a.

From the result of Figure 8c, there displayed sharp peaks. Therefore, it can be concluded that ordered structure corresponding to the second exothermic peak can be produced by the heating process. From the results of Figures $8 \mathrm{c}$ and $8 \mathrm{~d}$, ordered structure of L-PS was instable. It would be destroyed almost at the same time of its formation.

In some aspects, ordered structure and the second exothermic peak of L-PS is similar to that of polystyrene microspheres (micro-PS) proposed by $\mathrm{Fu}$ et al. ${ }^{11}$ But the difference is that according to Fu's argument, the ordered regions of micro-PS were imperfect and small size. The enthalpy of the second exothermic peak of micro-PS was only $6.4 \mathrm{~J} / \mathrm{g}$. Under the same conditions, the enthalpy of crystallization peak of iso-PS is $19.5 \mathrm{~J} / \mathrm{g}$ and the enthalpy of crystallization peak of syn-PS is $9.2 \mathrm{~J} / \mathrm{g} .{ }^{13}$ However, the enthalpy of the second exothermic peak of L-PS is $21.8 \mathrm{~J} / \mathrm{g}$. Usually, because of higher ordered degree, the enthalpy of crystallization peak of polymer should be higher than that of other instable ordered structure. Therefore,

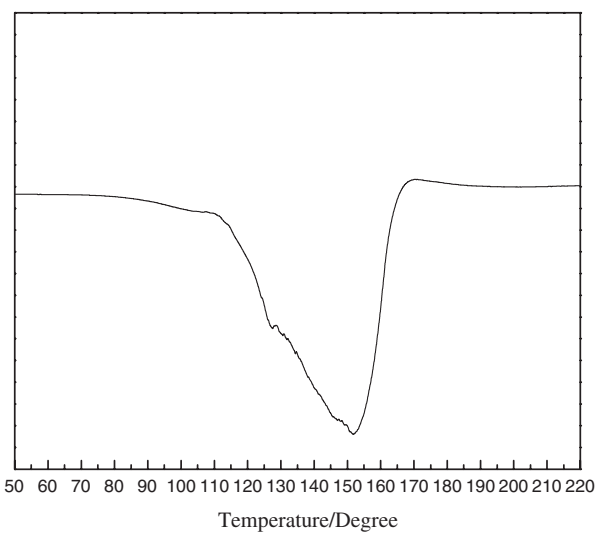

Figure 9. DSC thermograms of L-PS polymerized at $80^{\circ} \mathrm{C}$.

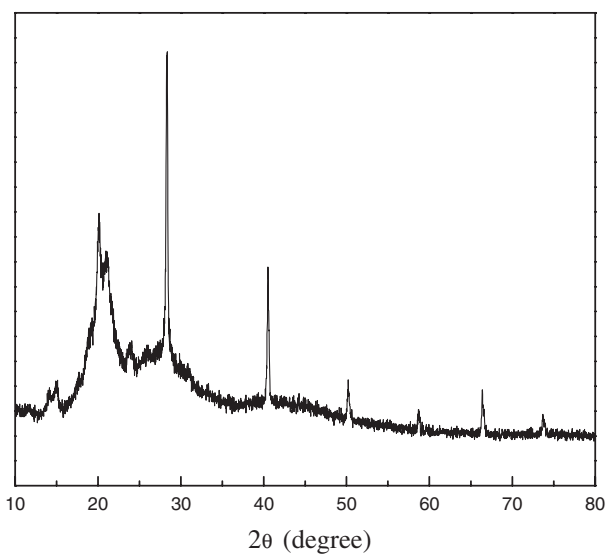

Figure 10. WAXD pattern of L-PS polymerized at $80^{\circ} \mathrm{C}$.

ordered regions of L-PS should be large size so that the second exothermic peak of L-PS had so high enthalpy.

When polymerization temperature of L-PS was increased to $80^{\circ} \mathrm{C}$, the effects of heating temperature on exothermic peaks were shown in Figure 9. The first exothermic peak and the second exothermic peak merged into a big exothermic peak whose highest peak was near $150{ }^{\circ} \mathrm{C}$. The enthalpy of the exothermic peak was $489 \mathrm{~J} / \mathrm{g}$. For the isotactic polystyrene, the values of the enthalpy of crystallization is $86.59 \mathrm{~J} / \mathrm{g}$ in Polymer handbook, ${ }^{14}$ and for syndiotactic polystyrene, the values of the enthalpy of crystallization is $53.2 \mathrm{~J} / \mathrm{g} .{ }^{15}$ Therefore, the enthalpy of the exothermic peak of L-PS is the most in known PS. The L-PS was heated to $150{ }^{\circ} \mathrm{C}$ and then quenched quickly into liquid nitrogen. It's WAXD pattern was shown in Figure 10. More evident peaks were found. For the sake of insurance, the same emulsion system without styrene was prepared and pure SDS was also investigated by WAXD. The result was shown in Figure 11. We can find that these sharp peaks in Figure 10 were not due to residual SDS. It means that the exothermic peak near $150{ }^{\circ} \mathrm{C}$ is main characteristic of L-PS, and L-PS can form some ordered structure during heating process. When the enthalpy of the exothermic peak near $150^{\circ} \mathrm{C}$ was bigger, the ordered structure of L-PS would be more obvious. 


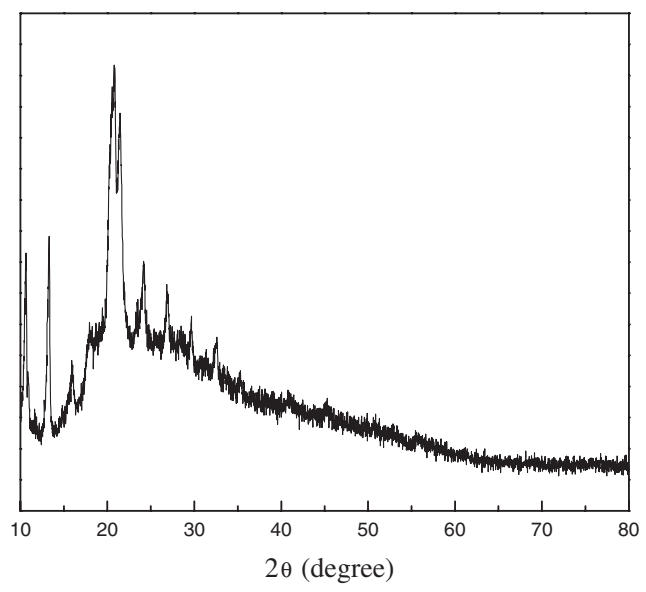

Figure 11. WAXD pattern of pure SDS.

As whole, L-PS was a kind of novel PS. Compared with crystalline PS, L-PS can form worse ordered structure under especial condition and the ordered regions were large.

What caused the especial polymer? We thought the reason was lamellar liquid-crystal template of polymerization. Styrene (St) is highly hydrophobic, and it must exist in hydrophobic space in polymerization system. ${ }^{16}$ In lamellar liquid-crystal of SDS, palisade layer offered many hydrophobic spaces which have lamellar morphology. Therefore, on the direction of thickness of L-PS, there were a large number of PS chain segments which had the same orientation and length. This is why L-PS has uniform thickness and smooth surface, just like Figure 12. The length of these molecular chain segments was thickness of L-PS. We thought that these chain segments were main chain of L-PS and easy to form ordered structure during heating process.

Based on results of our work, L-PS should have a novel molecular chain mode which had never been discovered in known PS before.

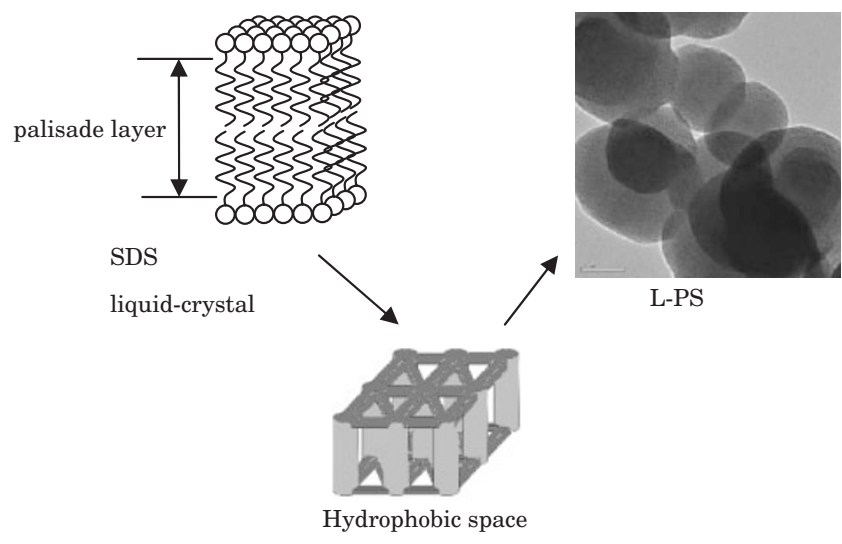

Figure 12. Schematic Representation of forming process of L-PS.

\section{REFERENCES}

1. H. Staudinger, Polymerization, Ber, 53, 1073 (1920).

2. H. M. James and E. Guth, J. Polym. Sci., 4, 153 (1949).

3. I. M. Ward, in "Mechanical Properties of Solid Polymers," 2nd, ed., John Wiley, New York, 1983, chapter 7 and 8.

4. R. K. Saini, I. W. Chiang, H. Peng, R. E. Smalley, W. E. Billups, R. H. Hauge, and J. L. Margrave, J. Am. Chem. Soc., 125, 3617 (2003).

5. J. Hu, T. W. Odom, and C. M. Lieber, Acc. Chem. Res., 32, 435 (1999).

6. S. Sun, C. B. Murray, D. Weller, L. Folks, and A. Moser, Science, 287, 1989 (2000)

7. S. A. Maier, P. G. Kik, H. A. Atwater, S. Meltzer, E. Harel, B. E. Koel, and A. A. G. Requicha, Nat. Mater., 2, 229 (2003).

8. "Emulsion Polymerization and Emulsion Polymer," P. A. Lovell and M. S. Elaasser, Ed., Wiley, Chichester, 1997.

9. J. J. Guo and X. Y. Li, Polym. J., 39 (11), 1120 (2007).

10. Y. M. Liu, X. H. He, X. F. Wang, and Y. W. Chen, Journal of Nanchang University, 2, 32 (2006).

11. W. H. Ming, J. Zhao, X. L. Lu, C. C. Wang, and S. K. Fu, Macromolecules, 29, 7678 (1996).

12. J. Mahr, J. Phys. Chem., 74, 2160 (1970).

13. H. X. Zeng, Z. P. Li, and H. Q. Wang, Acta Chim. Sinica, 57, 1055 (1999).

14. J. Brandup and E. H. Immergut, in "Polymer Handbook," Wiley Interscience, New York, 1989 p. V81-5.

Received: December 17, 2007 Accepted: March 11, 2008 Published: April 23, 2008
15. A. J. Pasztor, Jr., B. G. Landes, and P. J. Karjala, Thermochim. Acta 177, 187 (1991).

16. K. Takahashi and K. Nagai, Polymer, 37, 1257 (1996). 